\section{(c) (1) (8)}

OPEN ACCESS

Check for updates

Pakistan Institute of Community Ophthalmology, Hayatabad Medical Complex (Medical Teaching Institute), Peshawar-Pakistan ${ }^{2}$ Department of Ophthalmology, Hayatabad Medical Complex (Medical Teaching Institute), Peshawar-Pakistan

Address for correspondence: Mufarriq Shah

Pakistan Institute of Community Ophthalmology, Hayatabad Medical Complex (Medical Teaching Institute), Peshawar-Pakistan

E-mail:

mufarriq1@hotmail.com

Date Received:

September, $4^{\text {th }} 2021$

Date Revised:

October, $6^{\text {th }} 2021$

Date Accepted:

October, $10^{\text {th }} 2021$

This article may be cited as

Shah M, Khan MK. Management of low vision in people with myopic macular degeneration. J Postgrad Med Inst 2021;35(2):90-5. https://doi.org/10.54079/ jpmi.35.2.2766.

\title{
MANAGEMENT OF LOW VISION IN PEOPLE WITH MYOPIC MACULAR DEGENERATION
}

\author{
Mufarriq Shah ${ }^{\approx 1}$, Muhammad Tariq Khan ${ }^{2}$
}

\section{ABSTRACT}

Objective: To assess the effectiveness of correction of refractive error, use of contact lenses and low vision aids in visual rehabilitation of people with Myopic Macular Degeneration (MMD).

Methodology: This cross-sectional study included participants with MMD assessed for visual rehabilitation in a low vision clinic at Department of Ophthalmology Hayatabad Medical Complex, Peshawar, from June 2017 to June 2020. Data regarding distance and near visual acuities at the time of presentation with amount of myopia, best corrected visual acuity (VA) with glasses and contact lenses, VA with low vision devices and types of low vision devices prescribed were collected and analyzed. VA was recorded on Logarithm of the Minimum Angle of Resolution (LogMAR) chart. Data was analyzed using SPSS v.19.0.

Results: Out of 78 participants with MMD, $74.4 \%$ were male with mean age was $29.13 \pm 19.1$ years. About $22 \%$ had vision impairment with their own glasses, 19\% had severe impairment and 59\% had blindness. Mean spherical equivalent refractive error amongst participants was $-14.56 \pm 5.39 \mathrm{D}$ and $-12.52 \pm 6.64 \mathrm{D}$ in right and left eyes respectively. With optimum correction (glasses/contact lenses) in $41 \%$ of participants distance VA was improved to 6/18 (0.54 Log MAR) or better in the better-seeing eye. With low vision devices, mean distance visual acuity was enhanced to 0.18 Log MAR $(p<0.001)$.

Conclusion: Correction of myopia is very important in visual rehabilitation of people with MMD. Low vision aids can be successfully used to enhance the residual vision among people with MMD in order to improve life efficiency.

Keywords: Myopic Macular Degeneration (MMD); Blindness; Visual rehabilitation; Low vision devices.

\section{INTRODUCTION}

Globally, myopia is a major public health problem and contributes to the most common causes of irreversible vision loss. ${ }^{1-2}$ The prevalence of myopia reached epidemic in certain regions of the world, specifically in Asia. ${ }^{3-4}$ The prevalence of myopia is reported from $47.3 \%$ to $75.2 \%$ in those aged 20 to 29 years among Asian population. ${ }^{4}$ The world-wide increase in the number of people with myopia is anticipated to rise from 1.4 billion in 2000 to 4.75 billion in 2050 and high myopia (-5.0 D or less) from 163 million in 2000 to 938 million in $2050 .{ }^{1}$ The abnormal elongation of the eyeball in people with high myopia causes stretching and thinning of the retina, causing degeneration of the photoreceptors that leads to loss of central vision. 5,6 Ocular complications associated with myopia include cataract, glaucoma, retinal detachment and myopic macular degeneration (MMD). These complications cause irreversible vision loss in people with high myopia. $^{7}$
Myopic macular degeneration is reported as to be the first to third most frequent cause of irreversible blindness. ${ }^{7}$ In certain communities with high prevalence of myopia such as Japan and China, MMD is the leading cause of monocular blindness. ${ }^{8,9}$ Literature reported MMD as the second leading cause of irreversible blindness in Japan. ${ }^{8}$ MMD is contributing for $12.2 \%$ and $12.5 \%$ of vision impairment and blindness in Japan and in elderly Chinese population in Taiwan respectively. ${ }^{10}$ MMD is reported as two to six per cent of patients and one to five per cent of children with low vision in Australia. ${ }^{10,11}$ MMD has been found to contribute $14.5 \%$ of vision impairment in Pakistan. ${ }^{12}$

Vision impairment and blindness in the earlier stage of life due to MMD increases the lifetime economic burden on individual, family and society due to related functional disabilities and loss of independence. ${ }^{13,14}$ Provision of visual rehabilitation services could reduce the functional disabilities caused by MMD.,215 This study aimed to assess the effectiveness of optical correction and use of contact lenses and low vision de- 
vices in visual rehabilitation of people with myopic macular degeneration.

\section{MATERIAL AND METHODS}

This cross-sectional study was conducted at Department of Ophthalmology Hayatabad Medical Complex, Peshawar, Pakistan from June 2017 to June 2020. The study was approved by the Research and Ethics Committee of the Hayatabad Medical Complex Peshawar. The study included participants with low vision due to myopic macular degeneration (MMD). This study used the World Health Organization definition for low vision, "A person with low vision is one who has impairment of visual functioning even after treatment and/or standard refractive correction, and has a visual acuity of less than $6 / 18$ to light perception, or visual field of less than $10^{\circ}$ from the center of fixation but who uses, or is potentially able to use vision for the planning and/or execution of a task."16 The study enrolled 78 participants using non-probability consecutive sampling. The participants enrolled had MMD with visual acuity (VA) less than 6/18 in the better-seeing eye and without other ocular comorbidities such as glaucoma, age related macular degeneration, keratoconus were included in this study. These participants were evaluated for visual rehabilitation at low vision clinic.

Ocular examination of each participant was conducted by the retina specialist before referring to the low vision clinic. At the low vision clinic all participants underwent detailed ocular examinations including:

Detail history of each participant, his/ her family history, information about his/her visual difficulties such as seeing distant objects and problems in reading, and previous history of low vision assessment and use of any low vision devices were recorded.

Anterior and posterior segment ocular examination of each participant with MMD was conducted using slit-lamp bio-microscopy and indirect ophthalmoscopy after mydriasis to re-confirm diagnosis.

Distance visual acuity (VA) was measured using Logarithm of the Minimum Angle of Resolution (Log MAR) chart, designed by Bailey-Lovie, with 5 optotypes on each line. Log MAR Tumbling "E" chart was used for participants who could not read English. William Feinbloom chart for the partially sighted was utilized for people with very low visual acuity. VA was measured with the patient's own spectacles (if worn) at a distance of four meter on each eye separately. If not possible at a distance of four meter, then three, two or one meter distance was used when needed.

Near visual acuity was assessed using William Feinbloom chart for the partially sighted for reading and Bailey-Lovie chart for reading. Lea symbols for near visual acuity were used for illiterate people who want enhancement of near vision for tasks other than reading.

Retinoscopy was performed on all participants by an experienced optometrist. This was followed by subjective refraction, using standard techniques, of all participants for prescription of optical corrections for refractive errors. Spherical equivalent (SE) refraction was calculated as the algebraic sum of the sphere and half of the cylinder. Participants who were not willing to use glasses all the times and wanted contact lenses were evaluated if suitable for use of contact lenses. Participants with eye infections, conjunctivitis, blepharitis, keratitis, lacrimal tract obstruction, reduced corneal sensitivity, ptosis, chronic allergic were considered were not suitable for contact lenses. Assessment for contact lenses included keratometry reading, base curve determination for contact lenses and conversion of spectacle power to contact lenses using formula:

$$
\text { Contact lens power }=\frac{\text { Spectacle power }}{1-(\text { Back vecter power })(\text { Spectacle power })}
$$

Low vision devices were used for enhancing distance and near visual acuities. This include monocular and binocular telescopes of varying magnification, Flip-on reading cap with telescope, Ocutech bioptic telescope, Ocutech's Sight-Scope and filters for various wave lengths for distance vision; hand hold and stand magnifiers for near vision. Most of these low vision devices are available for assessment of low vision patients in tertiary care low vision clinics. However some advanced low vision devices such as Ocutech bioptic telescope, Ocutech Sight-Scope and Flip-on reading caps are not available in any other low vision clinic in Pakistan. One of the advantages of these advanced low vision devices is that these can be used for driving as well. Drivers with bioptic telescope have been licensed in many states in USA and Canada.

This study evaluated the presenting visual acuity in the better-seeing eye, the prescribed optical correction, the best corrected visual acuity in the better-seeing eye, and the visual acuity obtained with the recommended low vision device(s) for each participant. Visual acuity in the better-seeing eye was classified as: No impairment (6/6 to 6/18); Vision impairment (Less than 6/18 to 6/60); severe vision impairment (Less than 6/60 to 3/60) and blindness (Less than 3/60 to PL) as per WHO classification. ${ }^{17}$

SPSS (Statistical Package for Social Sciences) version 19 (IBM Corp, Armonk, NY, USA) was used for analysis. For statistical differences such as between visual acuity prior and with the provision of low vision devices, Mann-Whitney U-test was used and the data was presented descriptively as mean values and standard deviation.

\section{RESULTS}

A total of 78 consecutive people with myopic macular degeneration (MMD) assessed for visual rehabilitation. Participants were 
between age of 10 to 80 years (mean age was $29.13 \pm 19.1$ years). Male participants comprises more $(74.4 \%$; $n=58)$ than female (25.6\%; n=20). In our study 25 (32.1\%) people with MMD were younger than 16 years. The distribution of people with MMD by age and their distance visual acuity at presentation is shown in Table 1.

On presentation, 22\%, $19 \%$ and $59 \%$ had vision impairment, severe vision impairment and blindness respectively (using WHO low vision criteria) with their own glasses while no participant had visual acuity (VA) of 6/18 (0.54 Log. MAR) or better in the better-seeing eye. Mean VA at presentation was 1.26 Log MAR in better-seeing eyes.

Mean spherical equivalent refractive error amongst participants was $-14.56 \pm 5.39 \mathrm{D}$ and $-12.52 \pm 6.64 \mathrm{D}$ in right eyes and left eyes respectively. Overall, myopia among these people with MMD ranged from $-6.0 \mathrm{D}$ to $-28.00 \mathrm{D}$. In all participants, the means of cylindrical refractive errors in right eyes and left eyes were $1.27 \pm 1.59 \mathrm{D}$ and $0.73 \pm$ $1.20 \mathrm{D}$ respectively.

With optimum correction, mean improvement in distance visual acuity was 3 lines of Log. MAR on the Bailey-Lovie chart $(p=0.003)$. In $41 \%$ of participants distance VA was improved to 6/18 (0.54 Log. MAR) or better in the better-seeing eye and mean VA was improved to $0.83 \mathrm{Log}$. MAR with optimum correction (glasses/contact lenses). With the provision of low vision devices, 95\% achieved visual acuity 6/18 (0.54 Log. MAR) or better in the better-seeing eye while $5 \%$ $(n=4)$ remained in vision impairment category $(6 / 18>V A \geq 6 / 60)$. With low vision devices, mean distance visual acuity was enhanced to 0.18 Log MAR $(p<0.001)$. VA at presentation, VA with optimum correction and VA with low vision devices are given in Table 2.

Contact lenses were prescribed to 17 participants (10 female and 7 male) with high myopia (mean myopia of $-15.8 \mathrm{D}$ in right eyes and $-15.9 \mathrm{D}$ in left eyes). These participants did not want to wear spectacles. VA was significantly improved with contact lenses in these participants as compare to glasses $(p<0.001)$. With appropriate refraction their mean VA was $0.84 \pm 0.22 \mathrm{Log}$ MAR with glasses which was improved to 0 . $68 \pm 0.29$ Log. MAR with contact lenses. The estimated difference in means of VA with glasses and with contact lenses amongst these 17 participants was 0.15 Log MAR in better-seeing eyes. Of this group of 17 participants, $29.4 \%(n=5)$ achieved VA of 0.5 Log MAR while $17.6 \%(n=3)$ achieved 0.4 Log. MAR with contact lenses.

Amongst female participants with MMD, $60 \%(n=12)$ were in the blind category $(\mathrm{VA}<3 / 60)$ while amongst male participants with MMD 58.6\% ( $n=34)$ were in the blind category. With optimum correction (glasses/ contact lenses) only one female remained in the blind category. Comparisons of VA by gender on presentation and with optimum correction are given in Table 3.

Table 1: Distribution of people with MMD by age and their DVA at presentation $(n=78)$

\begin{tabular}{|c|c|c|c|c|}
\hline \multirow{2}{*}{ Age (Years) } & \multicolumn{3}{|c|}{ Categories of vision impairment } & Total \\
\cline { 2 - 5 } & $6 / 60 \leq \mathrm{VA}<6 / 18 \mathrm{n}(\%)$ & $3 / 60 \leq \mathrm{VA}<6 / 60 \mathrm{n}(\%)$ & $\mathrm{VA}<3 / 60 \mathrm{n}(\%)$ & $14(30.4)$ \\
\hline Less than 16 & $7(41.2)$ & $4(26.7)$ & $15(32.6)$ & $25(32.1)$ \\
\hline 16 to 29 & $4(23.5)$ & $4(26.7)$ & $7(15.2)$ & $11(14.1)$ \\
\hline 30 to 39 & $3(17.6)$ & $1(6.7)$ & $2(4.3)$ & $4(5.1)$ \\
\hline 40 to 49 & $1(5.9)$ & $1(6.7)$ & $1(6.7)$ & $1(1.3)$ \\
\hline 50 to 59 & 0 & $4(26.7)$ & $8(17.4)$ & $14(17.9)$ \\
\hline 60 years and older & $2(11.8)$ & $15(100)$ & $46(100)$ & $78(100)$ \\
\hline Total & $17(100)$ & & \\
\hline
\end{tabular}

Legends $\mathrm{MMD}=$ myopic macular degeneration; DVA= Distance visual acuity.

Table 2: Distribution of people with MMD according to DVA at presentation, DVA with correction of RE and DVA with LVDs $(\mathrm{n}=78)$

\begin{tabular}{|c|c|c|c|}
\hline VA Snellen (Log. MAR) & Presenting VA $n(\%)$ & VA With refraction $n(\%)$ & VA with LVDs $n(\%)$ \\
\hline $6 / 18(0.54)$ or better & $0(0)$ & $32(41.0)$ & $74(94.9)$ \\
\hline$<6 / 18$ to $6 / 60(<0.54-1.0)$ & $17(21.8)$ & $37(47.4)$ & $4(5.1)$ \\
\hline$<6 / 60$ to $3 / 60(<1.0-1.3)$ & $15(19.2)$ & $5(6.4)$ & $0(0)$ \\
\hline$<3 / 60(<1.3)$ & $46(59)$ & $4(5.1)$ & $0(0)$ \\
\hline Total & $78(100)$ & $78(100)$ & $78(100)$ \\
\hline
\end{tabular}

Legends MMD= myopic macular degeneration; DVA= Distance visual acuity; RE = Refractive errors; LVDs= Low vision devices 
Table 3: Gender-wise distribution of participants on the basis of presenting visual acuity and visual acuity with correction ( $\mathrm{n}=78$ )

\begin{tabular}{|c|c|c|c|c|}
\hline \multirow{2}{*}{ VA Snellen (Log MAR) } & \multicolumn{2}{|c|}{ VA on presentation } & \multicolumn{2}{|c|}{ VA with optimum correction } \\
\cline { 2 - 5 } & Male $n(\%)$ & Female $n(\%)$ & Male $n(\%)$ & $25(43.1)$ \\
\hline $6 / 18(0.54)$ or better & $0(0.0)$ & $0(0.0)$ & $26(44.8)$ & $11(35.0)$ \\
\hline$<6 / 18$ to $6 / 60(<0.54-1.0)$ & $12(20.7)$ & $5(25.0)$ & $4(6.9)$ & $1(5.0)$ \\
\hline$<6 / 60$ to 3/60 $(<1.0-1.3)$ & $12(20.7)$ & $3(15.0)$ & $3(5.2)$ & $1(5.0)$ \\
\hline$<3 / 60(<1.3)$ & $34(58.6)$ & $12(60.0)$ & $58(100)$ & $20(100)$ \\
\hline Total & $58(100)$ & $20(100)$ & \\
\hline
\end{tabular}

Table 4: Near visual acuity* at presentation \& with low vision aids $(\mathrm{n}=78)$

\begin{tabular}{|c|c|c|}
\hline Near VA & Presenting Near VA $n(\%)$ & Near VA with LVDs n(\%) \\
\hline 1 M or Better & $67(85.9)$ & $76(97.4)$ \\
\hline$<1 \mathrm{M}$ to 3.2M & $5(6.4)$ & $1(1.3)$ \\
\hline$<3.2 \mathrm{M}$ & $6(7.7)$ & $1(1.3)$ \\
\hline Total & $78(100)$ & $78(100)$ \\
\hline
\end{tabular}

*Near visual acuity without distance correction was noted

On presentation, $85.9 \%(\mathrm{n}=67)$ of participants with MMD had near visual acuity $1.0 \mathrm{M}$ (Newspaper print) or better in the better eye without distance correction. After prescribing low vision devices for reading, this number increased to $97.4 \%$ ( $n=76)$. Results of near visual acuity in better eyes of people with MMD at presentation without distance correction and near visual acuity with low vision devices are shown in Table 4.

For distance vision, spectacles were prescribed to all participants. Contact lenses were prescribed to 17 participants. Monocular telescopes were the most common low vision aid prescribed to $53.8 \%(n=42)$ of participants with MMD for enhancing distance VA. Most preferred low vision device for distance vision by people with MMD was VES Sport Ocutech Telescope but six participants could afford it. Hand Hold Magnifiers were prescribed to five participants, stand magnifiers to three participants and CCTV to two participants with MMD to meet their needs.

\section{DISCUSSION}

High myopia and associated ocular complications may lead to vision loss. ${ }^{8,9}$ Howev- er, findings from our study demonstrate that adequate optical correction and provision of glasses/contact lenses could improve functional vision of people with high myopia and resulting Myopic Macular Degeneration (MMD). The majority of people with MMD desired for low vision aids for distance vision tasks. Results from this study show that people with vision impairment due to MMD respond very well to magnification which can enable them to see faces, watch TV and even drive. Most of people with MMD have better vision for near task at a closed working distance.

In our study, the number of participants with vision impairment (VA less than 6/18 to 6/60) and blindness (VA less than 3/60 to $\mathrm{PL}$ ) was larger with their habitual vision at the time of presentation. The reason was inadequate optical correction. Similar results have been reported in other studies. 12,14,15 These findings demonstrate that referrals of people with MMD to low vision clinics are crucial for appropriate. However, only 78 people with MMD referred in a period of three years to the only tertiary care clinic in the province reflects that most of the people with MMD are not getting benefits from the available low vision rehabilitation services.
As reported in literature, findings from our study also indicate better improvement in vision with contact lenses in comparison to spectacles for people with high myopia. ${ }^{18}$ In spite of attempts to adequately correct high myopia, the best-corrected visual acuity in high myopic eyes was compromised with glasses as compare to contact lenses. One reason for poor vision with spectacles in high myopic eyes is minification of the retinal image by high power spectacle correction for high myopia. ${ }^{18,19}$ Functional vision could be enhanced with contact lenses in people with high myopia because lesser minification and larger field of view is achieved through contact lenses as compared to spectacles due to reduce vertex distance. Though the distance vision significantly improves with contact lenses as compare to glasses, the use of contact lenses for correction of high myopia is preferred due to enhanced cosmesis.

As reported in other studies from Pakistan, the number of female participants were lower in this study. ${ }^{14,19,20}$ Factors contributing to the lower number of female for visiting low vision services include low literacy ratio in females, the long distances involved for visiting the low vision clinic and refusal to accept spectacles and low vision devices due 
to cosmetic reasons.

Most attenders at our low vision clinic were interested to get benefit from simple low vision devices for distance vision. Findings from our study show that most of people with MMD were able to discern $1 \mathrm{M}$ (Newspaper print) or better without distance correction. High optical power of people with high myopia allow them enlargement of the retinal image by decreasing the distance between the eye and the near target while keeping it in focus. They simply need advice to take off their glasses and move the text closer to the eye till it becomes clear. Thus most of people with MMD could utilize their residual vision for reading purposes at a comparatively close working distance and do not need devices for reading purpose.

Two-third of our participants in the blind category due to MMD was in the productive period of life. These findings are consistent to another study carried out in Pakistan which reported MMD as a leading cause of vision loss in people under age 30 years. ${ }^{12}$ Similarly MMD has been identified as the third leading cause of blindness in children with vision impairment and blindness in $\mathrm{Pa}$ kistan. ${ }^{20}$ These findings indicate that there is an urgent need of provisions of visual rehabilitation services at primary and secondary levels eye care centers.

\section{- CONCLUSION}

This study suggests that optimum correction of myopia is very important in visual rehabilitation of people with Myopic Macular Degeneration (MMD). Low vision aids can be successfully used to enhance the residual vision among people with $\mathrm{MMD}$ in order to improve their efficiencies in activities of daily living. The less number of people with MMD in this study reflects low uptake of low vision rehabilitation services. Awareness about availability of low vision services and referrals of people with MMD to low vision clinics are crucial for appropriate management to reduce the functional disabilities caused by MMD.

\section{REFERENCES}

1. Fricke TR, Jong $M$, Naidoo KS, Sankaridurg P, Naduvilath TJ, Ho SM, et al. Global prevalence of visual impairment associated with myopic macular degeneration and temporal trends from 2000 through 2050: systematic review, meta-analysis and modelling. $\mathrm{Br} \mathrm{J}$ Ophthalmol. 2018;102(7):855-862.

2. Wong $Y-L$, Sabanayagam $C$, Ding $Y$, Wong $\mathrm{C}-\mathrm{W}$, Yeo AC-H, Cheung Y-B, et al. Prevalence, risk factors, and impact of myopic macular degeneration on visual impairment and functioning among adults in Singapore. Invest Ophthalmol Vis Sci. 2018;59(11):4603-4613.

3. Holden BA, Fricke TR, Wilson DA, Jong $M$, Naidoo KS, Sankaridurg $P$, et al. Global prevalence of myopia and high myopia and temporal trends from 2000 through 2050. Ophthalmology. 2016;123(5):1036-1042.

4. Wong YL, Saw SM. Epidemiology of pathologic myopia in Asia and worldwide. Asia Pac J Ophthalmol. 2016;5(6):394-402.

5. Holden B, Sankaridurg P, Smith E, Aller T, Jong M, He M. Myopia, an underrated global challenge to vision: where the current data takes us on myopia control. Eye. 2014;28(2):142-146.

6. Pan CW, Dirani M, Cheng CY, Wong TY, Saw SM. The age-specific prevalence of myopia in Asia: a meta-analysis. Optom Vis Sci. 2015;92(3):258-266.

7. Chui TY, Yap MK, Chan HH, Thibos LN. Retinal stretching limits peripheral visual acuity in myopia. Vision Res. 2005;45(5):593-605.

8. De Jong PT. Myopia: its historical contexts. $\mathrm{Br} J$ Ophthalmol. 2018;102(8):1021-1027.

9. Wong CW, Phua V, Lee SY, Wong TY,
Cheung CMG. Is choroidal or scleral thickness related to myopic macular degeneration? Invest Ophthalmol Vis Sci. 2017;58(2):907-913.

10. Wong TY, Ferreira A, Hughes $R$, Carter G. Mitchell P. Epidemiology and disease burden of pathologic myopia and myopic choroidal neovascularization: an evidence-based systematic review. Am J Ophthalmol. 2014;157(1):9-25. e12.

11. Iwase A, Araie M, Tomidokoro A, Yamamoto $T$, Shimizu $H$, Kitazawa $Y$, et al. Prevalence and causes of low vision and blindness in a Japanese adult population: the Tajimi Study. Ophthalmology. 2006;113(8):1354-1362. e1351.

12. Wu L, Sun X, Zhou X, Weng C. Causes and 3-year-incidence of blindness in Jing-An District, Shanghai, China 2001-2009. BMC Ophthalmol. 2011;11(1):10.

13. Yamada M, Hiratsuka $Y$, Roberts $C B$, Pezzullo ML, Yates K, Takano S, et al. Prevalence of visual impairment in the adult Japanese population by cause and severity and future projections. Ophthalmic Epidemiol. 2010;17(1):5057.

14. Chong MF, Jackson AJ, Wolffsohn JS, Bentley SA. An update on the characteristics of patients attending the Kooyong Low Vision Clinic. Clin Exp Optom. 2016;99(6):555-558.

15. Kalloniatis M, Johnston A. Visual characteristics of low vision children. Optom Vis Sci. 1990;67(1):38-48.

16. Shah M, Khan MD. Causes of low vision amongst the low-vision patients attending the low-vision clinic at Khyber institute of ophthalmic medical sciences (KIOMS), Hayatabad medical complex Peshawar, Pakistan. Vis Impair Res. 2004;6(2-3):89-97.

17. Frick KD. What the comprehensive economics of blindness and visual impairment can help us understand. Indian J Ophthalmol. 2012;60(5):406.

18. Naidoo KS, Fricke TR, Frick KD, Jong M, 
Naduvilath TJ, Resnikoff S, et al. Potential lost productivity resulting from the global burden of myopia: systematic review, meta-analysis, and modeling. Ophthalmology. 2019;126(3):338-346.
19. Sunness JS, El Annan J. Improvement of visual acuity by refraction in a low-vision population. Ophthalmology. 2010;117(7):1442-1446.

20. Chiang PP-C, Fenwick E, Cheung CMG,
Lamoureux EL. Public health impact of pathologic myopia. Pathologic myopia: Springer. 2014. p. 75-81.

\section{Author's Contribution}

MS conceptualized and designed the study, acquisition of data with analysis and interpretation of data, drafting of the article. MTK conceptualized, designed and final approval of the manuscript. Authors agree to be accountable for all aspects of the work in ensuring that questions related to the accuracy or integrity of any part of the work are appropriately investigated and resolved.

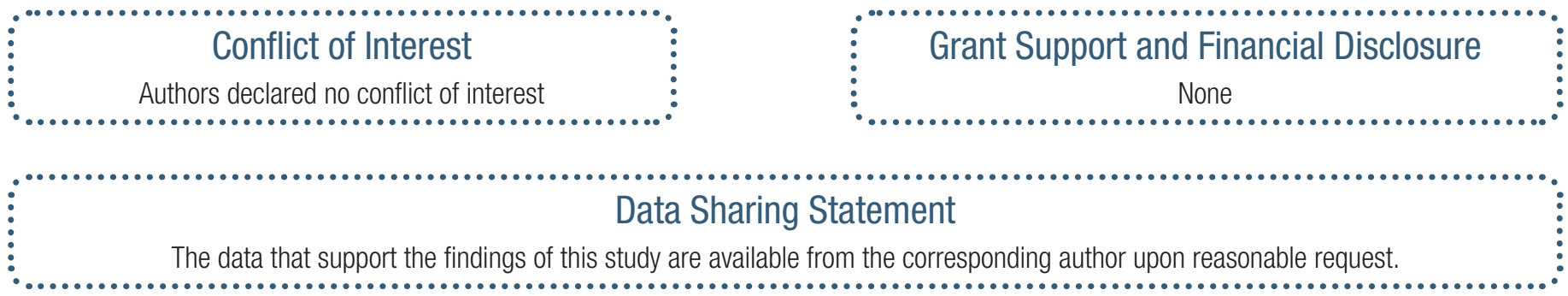

